\title{
Pseudomonas aeruginosa isolates from dental unit waterlines can be divided in two distinct groups, including one displaying phenotypes similar to isolates from cystic fibrosis patients
}

\author{
Myriam M. Ouellet ${ }^{1,2,3+}$, Annie Leduc ${ }^{4 \dagger}$, Christine Nadeau ${ }^{5}$, Jean Barbeau ${ }^{4+}$ and Steve J. Charette ${ }^{1,2,3+}$ \\ ${ }^{1}$ Centre de Recherche de I'Institut Universitaire de Cardiologie et de Pneumologie de Québec, Québec, OC, Canada \\ ${ }^{2}$ Institut de Biologie Intégrative et des Systèmes, Université Laval, Québec, OC, Canada \\ ${ }^{3}$ Département de Biochimie, de Microbiologie et de Bio-Informatique, Faculté des Sciences et de Génie, Université Laval, Québec, QC, Canada \\ ${ }^{4}$ Faculté de Médecine Dentaire, Université de Montréal, Montréal, OC, Canada \\ ${ }^{5}$ Faculté de Médecine Dentaire, Université Laval, Québec, QC, Canada
}

\section{Edited by:}

Evangelos Giamarellos-Bourboulis, University of Athens, Greece

Reviewed by:

Tamas Szakmany, Cardiff University, UK

Eirini Christaki, Agios Pavlos General Hospital, Greece

\section{*Correspondence:}

Jean Barbeau, Faculté de Médecine Dentaire, Université de Montréal, C.P. 6128, Succursale Centre-ville, Montréal, QC H3C 3J7, Canada e-mail: jean.barbeau@umontreal.ca

${ }^{t}$ These authors have contributed equally to this work.

\begin{abstract}
Pseudomonas aeruginosa displays broad genetic diversity, giving it an astonishing capacity to adapt to a variety of environments and to infect a wide range of hosts. While many $P$. aeruginosa isolates of various origins have been analyzed, isolates from cystic fibrosis (CF) patients have received the most attention. Less is known about the genetic and phenotypic diversity of $P$. aeruginosa isolates that colonize other environments where flourishing biofilms can be found. In the present study, $29 P$. aeruginosa isolates from dental unit waterlines and CF patients were collected and their genetic and phenotypes profiles were compared to determine whether environmental and clinical isolates are related. The isolates were first classified using the random amplified polymorphic DNA method. This made it possible to distribute the isolates into one clinical cluster and two environmental clusters. The isolates in the environmental cluster that were genetically closer to the clinical cluster also displayed phenotypes similar to the clinical isolates. The isolates from the second environmental cluster displayed opposite phenotypes, particularly an increased capacity to form biofilms. The isolates in this cluster were also the only ones harboring genes that encoded specific epimerases involved in the synthesis of lipopolysaccharides, which could explain their increased ability to form biofilms. In conclusion, the isolates from the dental unit waterlines could be distributed into two clusters, with some of the environmental isolates resembled the clinical isolates.
\end{abstract}

Keywords: Pseudomonas aeruginosa, cluster, RAPD, elastase, biofilm, Dictyostelium discoideum, cell lysis

\section{INTRODUCTION}

Pseudomonas aeruginosa is the most common cause of chronic lung infections affecting individuals with cystic fibrosis $(\mathrm{CF})$. CF is a multi-systemic disease, but the decline in respiratory function is the greatest cause of morbidity and mortality. Individuals suffering from CF produce excessive amounts of mucus in their lungs, which promotes the establishment of opportunistic pathogens that take advantage of a failing pulmonary physiology to establish chronic infections (Murray et al., 2007). Over $80 \%$ of CF patients at the age of 30 suffer from P. aeruginosa infections (Harrison, 2007). Once installed in the lungs, $P$. aeruginosa changes its behavior to adapt to its new environment by accumulating mutations and rearrangements in its genome (Kresse etal., 2003; Smith et al., 2006). The bacteria form a biofilm in the thick layer of abnormal mucus in the lungs of individuals with CF (Singh et al., 2000), enhancing the resistance of the bacteria to antibiotics and making their elimination by the immune system difficult or impossible (Murray et al., 2007).

Pseudomonas aeruginosa harbors a large genome $(\sim 6.5 \mathrm{Mb})$, and recent studies based on next generation sequencing (NGS) have revealed that there are variations between the genomes of clinical strains, confirming that this species can evolve and adapt during infections (Chung et al., 2012; Rau et al., 2012; Dettman et al., 2013; Marvig et al., 2013; Jeukens et al., 2014). Although NGS is a powerful approach, other more accessible genotyping methods can be used to classify $P$. aeruginosa isolates, including the random amplified polymorphic DNA (RAPD) technique, which has proven particularly useful in epidemiological studies involving $P$. aeruginosa isolates from CF patients (Mereghetti et al., 1998; Ortiz-Herrera et al., 2004; Fothergill etal., 2010b; Hafiane and Ravaoarinoro, 2011). RAPD generates reproducible and often distinctive sets of DNA fragments by subjecting genomic DNA to PCR primed by a single short oligonucleotide of arbitrary sequence. It is a rapid, cost-effective method that requires no prior genetic information on the target organism and that relies on low stringency priming sites for one or more arbitrarily chosen oligonucleotide primers on both strands of the DNA molecule.

Pseudomonas aeruginosa is an opportunistic pathogen that is found ubiquitously in water, soil, and plants. It can be also isolated from 2.9 to $50 \%$ of water samples collected from dental unit 
waterlines and from saliva ejector tubing (Barbeau et al., 1996, 1998). There is some evidence that dental unit waterlines colonized with bacteria, including $P$. aeruginosa, may be associated with infections (Martin, 1987; O’Donnell et al., 2011). Because of this, it is vital to identify the genetic and phenotypic features associated with CF infectious isolates from CF patients and to compare them to environmental isolates, particularly those from dental unit waterlines. Many phenotypic and genotypic studies of $P$. aeruginosa isolates have been reported (Deligianni et al., 2010; Fothergill et al., 2010a; Stehling et al., 2010; Bradbury et al., 2011; Hafiane and Ravaoarinoro, 2011; Lelong et al., 2011; Mowat et al., 2011; Chung et al., 2012; Hu et al., 2013; Workentine et al., 2013; Jeukens et al., 2014). However, most of these studies have focused on $\mathrm{CF}$ isolates without comparing them to environmental isolates or have compared CF isolates to environmental isolates with respect to a single aspect, such as the type three secretion system or the capacity of the bacteria to resist to predation by Dictyostelium discoideum amoebae (Bradbury et al., 2011; Hu et al., 2013). To our knowledge, no broad-based studies comparing the phenotypic and genotypic features of $P$. aeruginosa isolates from $\mathrm{CF}$ patients with those of isolates from dental unit waterlines have been conducted.

In the present study, we performed an RAPD analysis of $P$. aeruginosa isolates from dental unit waterlines and from young CF patients with lung infections. Our genotypic analysis enabled us to separate the isolates into three clusters. We also measured various phenotypic traits, including biofilm formation and resistance to amoeba predation to determine how these characteristics correlated with the RAPD clusters. While the clinical and environmental isolates were genetically distinct, some of the environmental isolates displayed the same array of phenotypes as the clinical isolates. However, the main outcome of our study was that there were two genetically and phenotypically distinct groups in the environmental isolates even though they all came from the same environment.

\section{MATERIALS AND METHODS}

\section{BACTERIA, AMOEBAE, AND CULTURE CONDITIONS}

Sixteen clinical and 13 environmental $P$. aeruginosa isolates were studied. The clinical isolates were collected from young CF patients aged 4 to 18 years of age with lung infections (Table 1). The environmental isolates were collected from dental unit waterlines at the Faculty of Dentistry of Université de Montréal. Table 2 lists the names and origins of the environmental isolates. API 20NE tests (BioMérieux) were used to confirm the identity of the $P$. aeruginosa isolates.

The $P$. aeruginosa isolates were cultivated in LB broth $(10 \mathrm{~g} / \mathrm{L}$ of peptone from casein, $5 \mathrm{~g} / \mathrm{L}$ of yeast extract, and $10 \mathrm{~g} / \mathrm{L}$ of $\mathrm{NaCl}$ in distilled water) or on LB agar (LB broth supplemented with $12 \mathrm{~g} / \mathrm{L}$ of Bacto agar) at $37^{\circ} \mathrm{C}$. When grown in broth medium, the cultures were shaken at $200 \mathrm{rpm}$.

We used the DH1-10 strain of D. discoideum (Cornillon et al., 2000). The cells were routinely cultivated in HL5 medium [ $14.3 \mathrm{~g} / \mathrm{L}$ of Bacto peptone (Oxoid L37), $7.15 \mathrm{~g} / \mathrm{L}$ of yeast extract, $18 \mathrm{~g} / \mathrm{L}$ of $\mathrm{D}$-(+)-monohydrate maltose, $0.641 \mathrm{~g} / \mathrm{L}$ of $\mathrm{Na}_{2} \mathrm{HPO}_{4} \cdot 2 \mathrm{H}_{2} \mathrm{O}$, and $0.490 \mathrm{~g} / \mathrm{L}$ of $\mathrm{KH}_{2} \mathrm{PO}_{4}$ in distilled water with a final $\mathrm{pH}$ of 6.5 ; Froquet et al., 2009] supplemented with $15 \mu \mathrm{g} / \mathrm{mL}$ of tetracycline.
Table 1 | Clinical Pseudomonas aeruginosa isolates from cystic fibrosis (CF) patients.

\begin{tabular}{lllll}
\hline $\begin{array}{l}\text { Isolate } \\
\text { number }\end{array}$ & Gender & Birth date & City ${ }^{\text {a }}$ & $\begin{array}{l}\text { Sample } \\
\text { date }\end{array}$ \\
\hline 279 & Female & $09-04-69$ & Montréal-Nord & $17-05-88$ \\
297 & Female & $25-08-80$ & Saint-Pie-de-Bagot & $01-06-88$ \\
358 & Female & $28-11-80$ & La Prairie & $04-10-88$ \\
359 & Female & $10-03-75$ & Répentigny & $04-10-88$ \\
392 & Male & $16-11-84$ & Saint-Roch-de-I'Achigan & $24-11-88$ \\
403 & Female & $19-04-74$ & Trois-Rivières & $10-01-89$ \\
E-500 & Female & $24-02-76$ & Mont-Laurier & $26-09-89$ \\
506 & Female & $26-08-75$ & Saint-Blaise & $10-10-89$ \\
$578-A$ & Male & $25-09-73$ & Montréal-Nord & $31-06-90$ \\
$578-B$ & Female & $15-06-75$ & Mont-Laurier & $31-06-90$ \\
585 & Female & $18-07-72$ & Saint-Jean sur Richelieu & $21-08-90$ \\
VD 171 & Female & $04-11-75$ & McMasterville & $27-10-87$ \\
VD 329 & Female & $04-11-75$ & McMasterville & $23-08-88$ \\
VD 706 & Female & $04-11-75$ & McMasterville & $17-10-89$ \\
VD 564 & Female & $04-11-75$ & McMasterville & $15-05-90$ \\
VD 609 & Female & $04-11-75$ & McMasterville & $05-12-90$
\end{tabular}

a The patients were all from cities in the Province of Quebec, Canada.

Table 2 | $\boldsymbol{P}$ aeruginosa isolates from dental unit waterlines.

\begin{tabular}{|c|c|c|}
\hline Dental clinic section & Section abbreviation & Dental unit number \\
\hline \multirow[t]{8}{*}{ Partial/fixed prosthesis } & PPF & 1 \\
\hline & & 2 \\
\hline & & 7 \\
\hline & & 13 \\
\hline & & 18 \\
\hline & & 19 \\
\hline & & 20 \\
\hline & & 21 \\
\hline \multirow[t]{2}{*}{ Emergency } & Urg & 5 \\
\hline & & 7 \\
\hline Orthodontics & Ortho & 1 \\
\hline \multirow[t]{2}{*}{ Surgery } & Chir & D-144 \\
\hline & & D-144 assistant \\
\hline
\end{tabular}

The amoebal cultures were grown at $21^{\circ} \mathrm{C}$ in $10-$ or $15-\mathrm{cm}-$ diameter polystyrene Petri dishes. The cultures were diluted twice a week in fresh medium to avoid confluence.

\section{RAPD}

Genomic DNA was extracted using a standard procedure (Smith et al., 1989; Wilson, 2001), with an additional RNase treatment (Schmidt et al., 1996), and was optimized for P. aeruginosa strains by including an additional 10 -min incubation period at $100^{\circ} \mathrm{C}$ 
to help lyse the cells at the beginning of DNA extraction. The quality of the genomic DNA was verified by $1 \%$ agarose gel electrophoresis in TBE (Tris-Borate-EDTA) buffer. Gels were stained with ethidium bromide and were camera-captured under ultraviolet light. The concentration of genomic DNA was calculated by measuring the optical density at $260 \mathrm{~nm}$, and the quality of the DNA was estimated using the $260 \mathrm{~nm} / 280 \mathrm{~nm}$ ratio and gel electrophoresis.

To select suitable candidate primers, 54 random sequence nanomer and decamer oligonucleotides were initially screened using two dental unit isolates and two clinical isolates (data not shown). Primers that generated a low number of distinct bands and at least two polymorphic DNA patterns with these isolates were then tested using the full panel of $29 P$. aeruginosa isolates. Three primers were selected: 910-07 $\left(5^{\prime}-\right.$ CCGCGGGAG-3'), 910-25 (5'-GCCCGGCAG-3'), and OPA-10 (5'-GTGATCGCAG-3').

Random amplified polymorphic DNA amplifications were performed in $25 \mu \mathrm{l}$ volumes. The amplification reaction mixtures contained $200 \mathrm{mM}$ each of dATP, dCTP, dGTP, and dTTP (Invitrogen), $1.2 \mu \mathrm{M}$ primer, 10 or $1 \mathrm{ng}$ of template DNA, and 2.5 units of Taq DNA polymerase (Perkin Elmer) in 10X Amplitaq Gold buffer (Perkin Elmer; $100 \mathrm{mM}$ Tris-HCl, pH 8.3, $500 \mathrm{mM}$ $\mathrm{KCl}, 15 \mathrm{mM} \mathrm{MgCl}_{2}$, and $0.01 \%$ gelatin) supplemented with $0.1 \%$ (v/v) Triton X-100. A negative control without template DNA was included in each experiment. The reaction mixture was overlaid with sterile mineral oil (Sigma-Aldrich) and was amplified in a DNA Thermal Cycler (Cetus; Perkin Elmer) programmed for one 7 -min denaturing pre-cycle at $94^{\circ} \mathrm{C}$ followed by 30 cycles of $1 \mathrm{~min}$ at $94^{\circ} \mathrm{C}, 1 \mathrm{~min}$ at $32^{\circ} \mathrm{C}$, and $1 \mathrm{~min}$ at $72^{\circ} \mathrm{C}$ followed by a final $7-\mathrm{min}$ elongation step at $72^{\circ} \mathrm{C}$. The amplification products, which made up the RAPD fingerprints, were analyzed by the electrophoresis of $10 \mu \mathrm{l}$ samples on $1.5 \%$ agarose gels run in TBE buffer and then stained with ethidium bromide. A 1-kb Plus DNA ladder molecular weight standard (Invitrogen) was included in each gel. The gels were camera-captured under ultraviolet light.

\section{ANALYSIS OF ISOLATE RELATEDNESS}

Computer-assisted analysis of the fingerprints was performed using the Taxotron package (Institut Pasteur), a set of programs for molecular systematics (RestrictoScan ${ }^{\circledR}$, RestrictoTyper ${ }^{\circledR}$, Adanson $^{\circledR}$, and Dendrograph ${ }^{\circledR}$ ). The derived molecular sizes (in base pairs) were used to compute distances (D) based on band sharing as the complement of the Dice coefficient: $D=1-\left(2 \mathrm{n}_{\mathrm{xy}} / \mathrm{n}_{\mathrm{x}}+\mathrm{n}_{\mathrm{y}}\right)$, where $\mathrm{n}_{\mathrm{xy}}=$ the number of bands shared by isolates $\mathrm{x}$ and $\mathrm{y}$, and $\mathrm{n}_{\mathrm{x}}$ and $\mathrm{n}_{\mathrm{y}}=$ the number of bands scored for each individual. In the pairwise comparison to match co-migrating fragment positions between pairs of RAPD fingerprints, a match was recorded if the normalized molecular size of the first amplicon was within a $\pm 3 \%$ window of the molecular size of the second amplicon. A matrix combining all the distances was generated, and a cluster analysis was performed using the unweighted pair-group method with mathematical averages. A dendrogram was then constructed. To generate a dendrogram based on the combined fingerprints obtained with all three primers, the information contained in the three band data files was combined in a new band data file, which was then processed as described above.

\section{SLIME PRODUCTION}

We evaluated slime production as already described (Podbielska et al., 2010). Briefly, we inoculated Congo Red Agar (Brain Heart Infusion, BD supplemented with 5\% (w/v) filter-sterilized sucrose, $0.08 \%(w / v)$ Congo Red) directly with frozen isolate stocks. The plates were incubated at $37^{\circ} \mathrm{C}$ for $24 \mathrm{~h}$ and then at room temperature for $48 \mathrm{~h}$. We noted the morphologies and characteristics of each isolate and used PA01 and PA14 as controls. Colonies with a mucoid phenotype were scored ++ , those with a slightly shiny phenotype were scored + , and flat colonies were scored 0 .

\section{BIOFILM FORMATION}

Frozen stocks were inoculated on Tryptone soy agar (BD) plates supplemented with $5 \%(\mathrm{v} / \mathrm{v})$ sheep blood (Oxoid), which were incubated at $37^{\circ} \mathrm{C}$ for $24 \mathrm{~h}$. Tryptic soy broth (BD) cultures were inoculated with the precultures and incubated overnight at $37^{\circ} \mathrm{C}$ without shaking.

The optical densities at $600 \mathrm{~nm}$ of the cultures were measured. The cultures were centrifuged, and the pellets were washed twice with potassium phosphate buffer (PBS, $50 \mathrm{mM}, \mathrm{pH}$ 7.2) containing $150 \mathrm{mM} \mathrm{NaCl}$. The pellets were suspended in $1.5 \%(\mathrm{w} / \mathrm{v})$ proteose-peptone (BD) in PBS at an optical density of 0.15 at $600 \mathrm{~nm}$. The suspensions were serially diluted $\left(10^{-4}\right)$ in the following autoclaved culture medium to measure biofilm formation (in L): $17 \mathrm{~g}$ of Tryticase peptone (BBL), $3 \mathrm{~g}$ of yeast extract (BBL), $5 \mathrm{~g}$ of $\mathrm{NaCl}$ (Fisher Scientific), and $2.5 \mathrm{~g}$ of sodium phosphate monobasic monohydrate (Acros Organics) to which $0.5 \%(\mathrm{w} / \mathrm{v})$ filter-sterilized glucose was added. Aliquots $(100 \mu \mathrm{L})$ of the diluted bacterial suspension were placed in 96-well microplates (16 wells for each dilution; Corning, Costar). The microplates were incubated at $30^{\circ} \mathrm{C}$ without shaking for $24 \mathrm{~h}$. Uninoculated medium was used as a negative control. The optical density at $620 \mathrm{~nm}$ was measured using a microplate reader (AD340; Beckman Coulter). The wells were then washed twice with sterile $0.85 \%$ (w/v) $\mathrm{NaCl}$, dried, stained for 15 min with gram crystal violet (BD), rinsed under tap water, and dried again. The stain was decolorized using $200 \mu \mathrm{L}$ of the following destaining solution: $15 \%(\mathrm{v} / \mathrm{v})$ acetic acid and $10 \%(\mathrm{v} / \mathrm{v})$ methanol. The optical density at $570 \mathrm{~nm}$ was measured using a microplate reader. Optical densities at $620 \mathrm{~nm}$ under 0.1 were considered as no growth. The results are expressed as optical densities at $570 \mathrm{~nm}$ using a $10^{-4}$ inoculum.

\section{ELASTASE ASSAY}

The isolates were grown for $24 \mathrm{~h}$ in $\mathrm{LB}$ at $37^{\circ} \mathrm{C}$ with shaking, and the optical densities at $600 \mathrm{~nm}$ were measured (Spectronic Biomate 3; Thermo Electron). The cultures were centrifuged, and the elastase activities in the culture supernatants were determined using a previously published protocol (Rust et al., 1994). In brief, $10 \mathrm{mg}$ of elastin-Congo red (Sigma-Aldrich) was added to $1 \mathrm{ml}$ of concentrated culture supernatant diluted with $2 \mathrm{ml}$ of the following buffer: $30 \mathrm{mM}$ Tris, $\mathrm{pH} 7.2,1 \mathrm{mM} \mathrm{CaCl}_{2}$. The mixtures were incubated for $5 \mathrm{~h}$ at $37^{\circ} \mathrm{C}$ with shaking. The results are expressed as the $495 \mathrm{~nm} / 600 \mathrm{~nm}$ ratio.

\section{PREDATION ASSAY}

The assay was used to determine the resistance of the isolates to amoebal predation (Filion and Charette, 2014). The assay was 
performed in triplicate with each isolate. The bacterial precultures in LB broth in snapcap tubes were incubated overnight at $37^{\circ} \mathrm{C}$ with a shaking at $200 \mathrm{rpm}$. The precultures $(300 \mu \mathrm{L})$ were then gently spread on $10-\mathrm{cm}$ Petri dishes containing $35 \mathrm{ml}$ of autoclaved SM 1/10 agar medium ( $1 \mathrm{~g} / \mathrm{L}$ of bactopeptone, $0.1 \mathrm{~g} / \mathrm{L}$ of yeast extract, $0.22 \mathrm{~g} / \mathrm{L}$ of $\mathrm{KH}_{2} \mathrm{PO}_{4}, 0.1 \mathrm{~g} / \mathrm{L}$ of $\mathrm{K}_{2} \mathrm{HPO}_{4}, 0.1 \mathrm{~g} / \mathrm{L}$ of $\mathrm{MgSO}_{4} \cdot 7 \mathrm{H}_{2} \mathrm{O}$, and $20 \mathrm{~g} / \mathrm{L}$ of Bacto agar in $950 \mathrm{~mL}$ of distilled water supplemented with $50 \mathrm{ml}$ of $2 \mathrm{~g} / 100 \mathrm{~mL}$ of filter-sterilized glucose; Filion and Charette, 2014) to obtain uniform bacterial lawns. The Petri dishes were then dried in a laminar flow hood and various concentrations of DH1-10 amoeba from cultures with no more than $60 \%$ confluence were suspended in HL5 medium and $5-\mu \mathrm{L}$ drops were deposited on the bacterial lawns. The concentrations of the amoebae ranged from 0 to 50,000 cells $/ 5 \mu \mathrm{L}$. Once the drops had dried, the Petri dishes were incubated at $21^{\circ} \mathrm{C}$ and were examined for phagocytic plaques after 7 days.

\section{LYSIS ASSAY}

The lysis assay was used to determine whether the $P$. aeruginosa isolates secreted products that can lyse $D$. discoideum cells (Cosson et al., 2002). The lysis assay was performed in sterile polystyrene 24 -well plates. HL5 medium $(500 \mu \mathrm{L})$ containing 500,000 D. discoideum cells from cultures at no more than $60 \%$ confluence was placed in each well, and the plates were incubated for $1 \mathrm{~h}$ at $21^{\circ} \mathrm{C}$.

The day before the experiments, bacterial precultures of each $P$. aeruginosa isolate were incubated in $2 \mathrm{~mL}$ of LB broth for $24 \mathrm{~h}$ at $37^{\circ} \mathrm{C}$ with shaking at $200 \mathrm{rpm}$. The bacterial precultures were then centrifuged at $3220 \mathrm{~g}$ for $10 \mathrm{~min}$ and the supernatants were collected and filter sterilized using $0.20-\mu \mathrm{m}$ polyethersulfone filters attached to sterile syringes. The HL5 medium in the wells of the polystyrene 24 -well plates was gently removed and was carefully replaced by filtered supernatants from the $P$. aeruginosa isolates. The wells were photo-captured at 0,5 , and $10 \mathrm{~min}$ using a Moticam Pro 252A camera and Motic Images Advanced 3.2 software (Motic). The procedure was repeated for every supernatant from four different experiments. The disappearance of amoebal refringence is associated with lysis (Cosson et al., 2002). The percentage of cell lysis was determined after a $10 \mathrm{~min}$ exposure to the bacterial supernatants. The PAO1 strain was used as a positive control (Cosson et al., 2002).

\section{CLONING THE 600-bp BAND SPECIFIC FOR CLUSTER III ISOLATES}

Random amplified polymorphic DNA using primer OPA-10 was performed on PPF 2 and PPF 7 (cluster III) with parameters identical to those described above, with the following differences. Taq polymerase and standard buffer (M0273L; NEB) were used. No mineral oil was added to the PCR tubes. Hot start PCRs were performed using the following protocol: $94^{\circ} \mathrm{C}$ when adding the enzyme, followed by 30 cycles of $1 \mathrm{~min}$ at $94^{\circ} \mathrm{C}, 1 \mathrm{~min}$ at $29^{\circ} \mathrm{C}, 1 \mathrm{~min}$ at $72^{\circ} \mathrm{C}$, and a final 7 -min elongation step at $72^{\circ} \mathrm{C}$. The resulting 600 -bp RAPD amplification product (OPA10) was cloned into plasmid $\mathrm{pCR}^{\mathrm{TM}}$-Blunt II TOPO ${ }^{\circledR}$ (Invitrogen) according to manufacturer's protocol.

Insert sequencing was performed at the IRIC Genomic Platform (Université de Montréal). A blast analysis (see Results section for details) revealed that the 600-bp fragment corresponds to a region overlapping the $w b j C$ and $w b j D$ genes, both of which code for epimerases.

\section{Gyrase B AND wbjC-wbjD PCR GENOTYPING}

One primer pair was designed for the gyrase $\mathrm{B}$ gene used as a positive PCR control (GyrB-F1: 5'-GAG TAC CTG AAC ACC AAC AAG A-3' + GyrB-R1: 5'-AGT CAC CCT CCA CGA TGT A-3'). It generated a 604-bp amplicon. Another primer pair was designed to target a region overlapping the $w b j C$ and $w b j D$ genes (EPI-F2: 5'-CCT GGA TGG ACT CAT GAC ATT AC-3' + EPI-R2: 5'-CAT GAC CCT AGA CAA GCG AAT AA-3'). It generated a 212-bp amplicon. The primers were synthesized by Life Technologies.

Either purified bacterial DNA (10 ng, see the RAPD section) or $3 \mu \mathrm{l}$ of diluted cell lysates (1:10) from single colonies suspended in $20 \mu \mathrm{l}$ of SWL buffer [ $50 \mathrm{mM} \mathrm{KCl,} 2.5 \mathrm{mM} \mathrm{MgCl}_{2}, 10 \mathrm{mM}$ Tris, $\mathrm{pH} 8.3$, and $0.45 \%(\mathrm{v} / \mathrm{v}) \mathrm{NP}-40$ and Tween 20] were used for the PCR templates depending on the availability of the material. In the case of the lysates, samples were heated at $95^{\circ} \mathrm{C}$ for $5 \mathrm{~min}$ in a thermocycler to complete the lysis.

The conditions described for the RAPD procedure were used except that the gelatin and Triton $\mathrm{X}-100$ were omitted. The hot start protocol was as follows: an initial 2.5 -min incubation at $95^{\circ} \mathrm{C}$ followed by 30 cycles of $30 \mathrm{~s}$ at $95^{\circ} \mathrm{C}, 30 \mathrm{~s}$ at $55^{\circ} \mathrm{C}$, and $1 \mathrm{~min}$ at $72^{\circ} \mathrm{C}$, followed by a final 7 -min elongation step at $72^{\circ} \mathrm{C}$. The PCR was performed twice for each isolate.

\section{RESULTS}

\section{THE RAPD ASSAY SEPARATED THE $P$. aeruginosa ISOLATES INTO THREE CLUSTERS}

Twenty-nine $P$. aeruginosa isolates [16 from CF patients (clinical) and 13 from dental unit waterlines (environmental)] were collected and compared (Tables 1 and 2). Three random probes were chosen for the RAPD assay in order to genotype the isolates and generate a dendrogram (see Materials and Methods). The RAPD assay easily discriminated the $P$. aeruginosa isolates into clinical and environmental clusters (Figure 1). Three clusters (clusters I, II, and III) were generated using a genetic distance of $60 \%$ as a threshold. At this similarity level, both the clinical and environmental isolates clearly clustered into distinct groups. The intracluster similarity for each cluster was approximately $65 \%$ or greater.

All the clinical isolates belonged to cluster I, except for isolates 506 and 578-B, which belonged to cluster II. The VD series $P$. aeruginosa isolates, which were isolated from the same patient over a 3-year period, shared $90 \%$ similarity. Isolate 297 , which was isolated from an unrelated patient, shared strong genetic similarity (90\%) with the VD series isolates. The environmental isolates were placed in clusters II and III even though they were all isolated in the same building and, as such, from the same municipal water supply. No environmental isolates were included in cluster I. Based on this clear genetic segregation of the isolates, we hypothesized that some phenotypic characteristics associated with virulence might also be distinctively associated with the three clusters.

\section{PHENOTYPES ASSOCIATED WITH THE CLUSTERS}

The aspects of colony isolates were recorded. Three different phenotypes were observed. Colonies of some isolates had a mucoid 


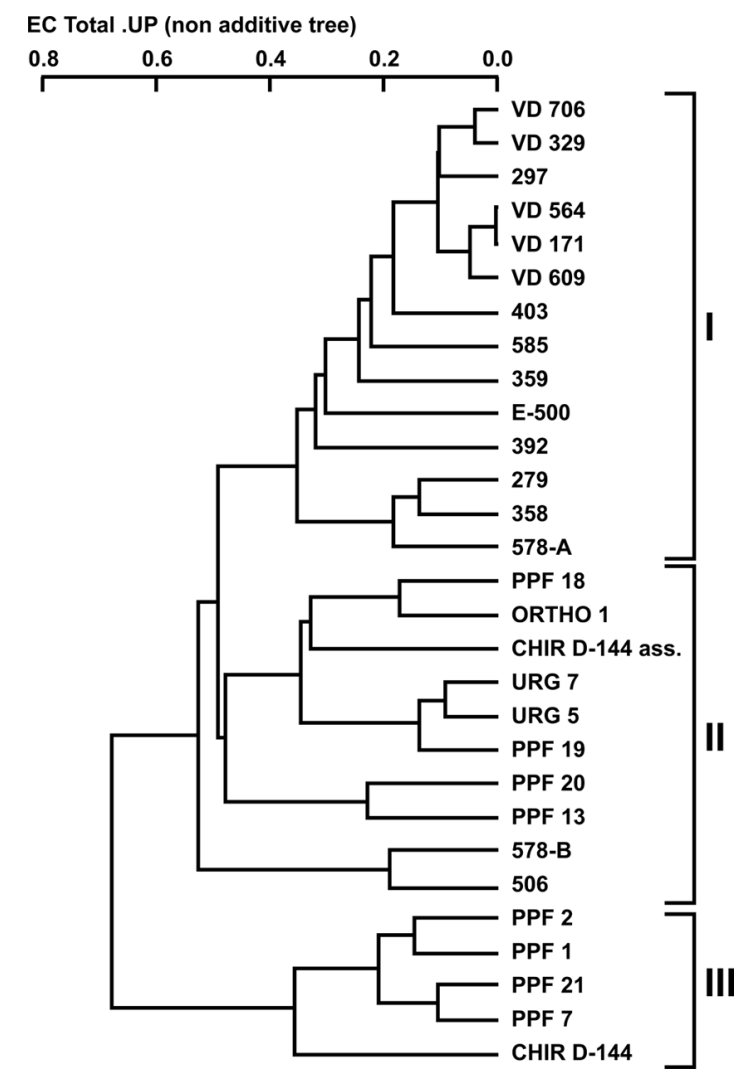

FIGURE 1 | Dendrogram of the combined random amplified polymorphic DNA (RAPD) fingerprints of Pseudomonas aeruginosa clinical and dental unit waterline isolates using the 910-25, 910-07, and OPA-10 primers.

phenotype (Table 3). These isolates were only found in cluster I (clinical isolates) but not all the clinical isolates presented a mucoid phenotype. Some isolates of cluster I had a slightly shiny phenotype, which was also observed with most isolates in cluster II. Lastly, all the isolates in cluster III had a flat colony phenotype.

The capacity to form biofilms was evaluated in polystyrene microplates using a crystal violet assay. When the isolates were compared based solely on their clinical or environmental origin, no significant differences were noted, although the environmental isolates had a slight tendency to produce more biofilm. However, significant differences were observed when the capacity of the isolates in the three clusters to form biofilms was evaluated. Cluster III included isolates that formed significantly more biofilm than those in clusters I $(p=0.042)$ and II $(p=0.012$; Figure 2$)$. Elastase production also discriminated cluster III from clusters I and II ( $p=0.034$; Figure 3). No differences were noted between cluster I and II in regards to biofilm formation and elastase activity.

THE INTERACTION OF $P$. aeruginosa WITH AMOEBAE WAS PARTIALLY ASSOCIATED WITH THE CLUSTERS

The $D$. discoideum predation assay could not distinguish between the clinical and environmental isolates or the clusters. (Figure 4; Table 4). Two clinical isolates and one environmental isolate of
Table 3 | Colony appearance of the $P$ aeruginosa isolates.

\begin{tabular}{|c|c|c|}
\hline$P$ aeruginosa isolate & Cluster & Colony phenotype \\
\hline VD 706 & I & ++ \\
\hline VD 329 & I & ++ \\
\hline 297 & I & + \\
\hline VD 564 & I & + \\
\hline VD 171 & 1 & ++ \\
\hline VD 609 & 1 & ++ \\
\hline 403 & 1 & + \\
\hline 585 & । & + \\
\hline 359 & 1 & ++ \\
\hline$E-500$ & I & 0 \\
\hline 392 & 1 & + \\
\hline 279 & I & ++ \\
\hline 358 & I & 0 \\
\hline $578-A$ & I & ++ \\
\hline PPF 18 & $\|$ & + \\
\hline Ortho 1 & ॥ & + \\
\hline Chir D-144 assistant & ॥ & + \\
\hline Urg 7 & ॥ & + \\
\hline Urg 5 & $\|$ & + \\
\hline PPF 19 & ॥ & + \\
\hline PPF 20 & ॥ & + \\
\hline PPF 13 & ॥ & + \\
\hline $578-B$ & ॥ & 0 \\
\hline 506 & $\|$ & + \\
\hline PPF2 & III & 0 \\
\hline PPF1 & III & 0 \\
\hline PPF21 & III & 0 \\
\hline PPF7 & III & 0 \\
\hline Chir D-144 & III & 0 \\
\hline
\end{tabular}

Colonies with a mucoid phenotype were scored ++ , those with a slightly shiny phenotype were scored + , and flat colonies were scored zero.

the 29 tested were not resistant to amoebal predation. All the other isolates were highly resistant to predation. Only the highest concentration of $D$. discoideum cells was able to produce phagocytic plaques in the bacterial lawns of the majority of the isolates. The three most permissive isolates (392, 578-B, and PPF7) allowed the growth of $D$. discoideum when as few as 5 to 50 amoebal cells were deposited on the bacterial lawns. None of the three permissive isolates belonged to a same cluster. Clinical isolates 392 and 578-B belonged to cluster I and II, respectively, and environmental isolate PPF7 belonged to cluster III. There was thus no correlation between the capacity of the $P$. aeruginosa isolates to resist amoebae predation and their RAPD cluster.

The ability to lyse amoebae differentiated between clinical and environmental isolates and between clusters (Figure 5; Table 5). Isolates from clusters I and II were either able to lyse amoebal cells or were variable in their ability. While some isolates lysed 


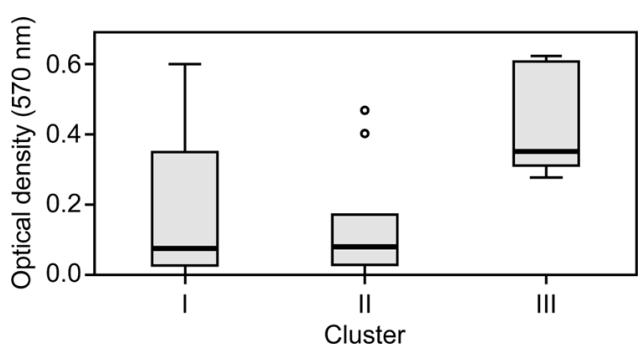

FIGURE 2 | Boxplot of biofilm-forming capacity. Isolates in cluster III produced significantly more biofilm than those in clusters I and II. Kruskal-Wallis with independent variables.

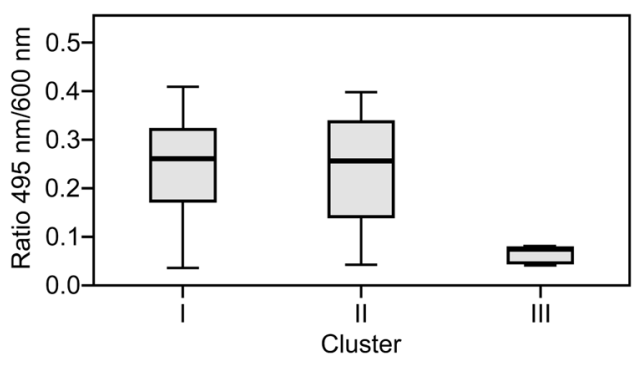

FIGURE 3 | Boxplot of elastase production. Isolates in cluster III produced significantly less elastase than those in clusters I and II. Kruskal-Wallis with independent variables.

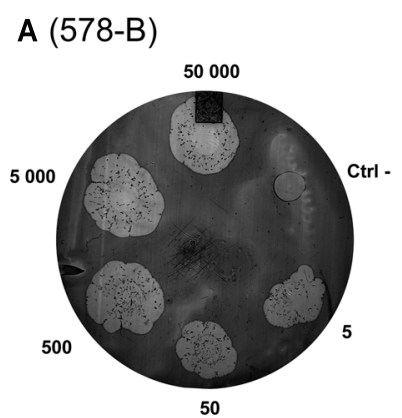

B (PPF 2)

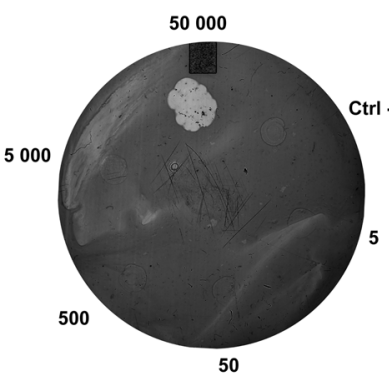

FIGURE 4 | Example of results obtained with the amoeba predation assay. Various quantities of amoebae were deposited on P. aeruginosa lawns (5 to 50,000 amoebae per spot). After a 7-day incubation, phagocytic plaques were visible where amoebae fed on the bacteria. The growth of the amoebae fed on the lawns of two isolates is illustrated: (A) Isolate 578-B is sensitive to predation; (B) Isolate PPF 2 is resistant to predation. The negative control (Ctrl-) was HL5 medium without amoeba.

amoebal cells, others did not and, for some isolates, the ability was highly variable from one experiment to another (Table 5). Variability was highest in cluster I. The lysis assay performed on the environmental isolates separated them into two groups: isolates that lysed amoebal cells (cluster II) and isolates that did not (cluster III; Figure 5; Table 5). In cluster II, the majority of the isolates were able to lyse amoebal cells, with the exception of Ortho1, 506, and urg5, which had a variable lytic phenotype, and $578-\mathrm{B}$, which displayed no lytic activity. No isolates in cluster III were able to lyse amoebal cells (Table 5).
Table 4 | Results of the amoeba predation assay.

\begin{tabular}{|c|c|c|}
\hline$P$ aeruginosa isolate & Cluster & Average $^{\mathrm{a}}$ \\
\hline VD 706 & 1 & 1.0 \\
\hline VD 329 & I & 0.7 \\
\hline 297 & 1 & 1.0 \\
\hline VD 564 & 1 & 1.0 \\
\hline VD 171 & 1 & 1.0 \\
\hline VD 609 & I & 1.0 \\
\hline 403 & I & 1.0 \\
\hline 585 & 1 & 2.0 \\
\hline 359 & 1 & 0.7 \\
\hline$E-500$ & 1 & 1.3 \\
\hline 392 & 1 & 5.0 \\
\hline 279 & I & 1.0 \\
\hline 358 & I & 2.3 \\
\hline $578-A$ & I & 1.3 \\
\hline PPF 18 & ॥ & 1.0 \\
\hline Ortho 1 & ॥ & 1.0 \\
\hline Chir D-144 assistant & ॥ & 1.0 \\
\hline Urg 7 & II & 1.0 \\
\hline Urg 5 & II & 1.0 \\
\hline PPF 19 & ॥ & 1.0 \\
\hline PPF 20 & $\|$ & 1.0 \\
\hline PPF 13 & II & 1.0 \\
\hline $578-B$ & ॥ & 5.0 \\
\hline 506 & II & 1.0 \\
\hline PPF2 & III & 1.0 \\
\hline PPF1 & III & 1.7 \\
\hline PPF21 & III & 1.3 \\
\hline PPF7 & III & 3.7 \\
\hline Chir D-144 & III & 1.7 \\
\hline
\end{tabular}

The values are the average of three independent experiments.

${ }^{a} A$ value is associated with the number of amoebae required to produce a phagocytic plaque in the bacterial lawn (see Figure 4): (1) phagocytic plaques appear with 50,000 amoebae; (2) phagocytic plaques appear with 5,000 amoebae; (3) phagocytic plaques appear with 500 amoebae; (4) phagocytic plaques appear with 50 amoebae; and (5) phagocytic plaques appear with five amoebae. The value is zero when the isolate is totally resistant to predation by the amoebae (i.e., no observable phagocytic plaque).

\section{GENES ENCODING EPIMERASES SPECIFIC TO CLUSTER III}

As illustrated by Table 6, which presents a summary of the phenotypic results obtained in this study, it appeared that $P$. aeruginosa isolates from the cluster III are those with the most distinctive phenotypes compared to the two other clusters even if isolates in cluster I displayed important variability for some of the phenotypes analyzed. In RAPD, an intense band with the OPA-10 primer was observed at $600 \mathrm{bp}$ for cluster III isolates (Figure 6). With the objective to have molecular hints on the distinctive characteristics of the cluster III, the intense 600-bp band specific to this cluster obtained in RAPD using the OPA-10 
A

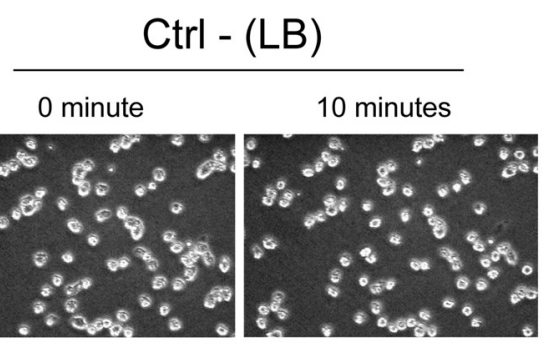

C

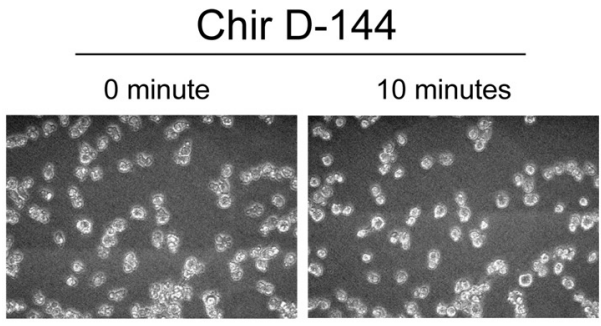

B

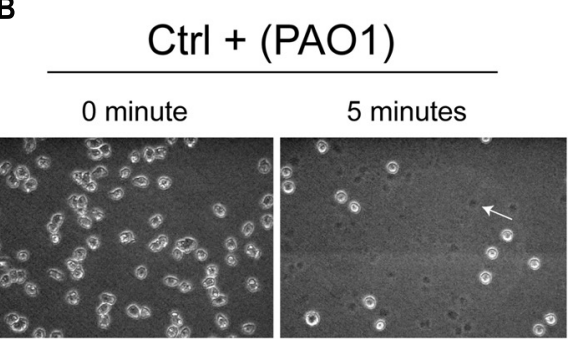

D

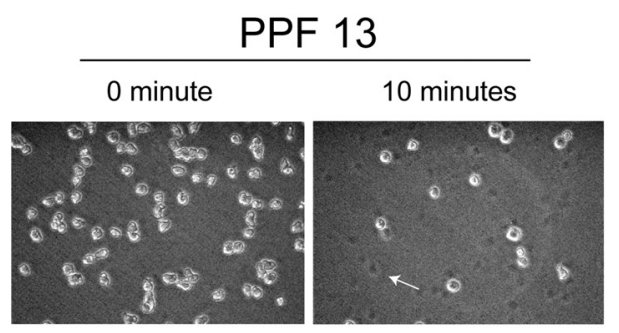

FIGURE 5 | Lysis of Dictyostelium discoideum cells exposed to $\boldsymbol{P}$ aeruginosa culture supernatants. $P$. aeruginosa culture supernatant in contact with amoebae grown in the wells of 24-well plates. The amoebae were overlain with $P$. aeruginosa culture supernatants, and images were acquired at 0,5 , and $10 \mathrm{~min}$. The images taken at each time correspond to the same field. (A) D. discoideum cultures overlain with fresh culture medium were used as negative controls. No cell lysis was observed. (B) PAO1 culture supernatants were used as positive controls (Cosson et al., 2002). A large number of $D$. discoideum cells were already lysed after 5 min. (C) Example of an isolate (Chir D-144) that was unable to lyse amoebae. (D) Example of an isolate (PPF 13) that caused marked cell lysis. Dead cells left a dark spot (arrow). primer was purified, cloned in a vector and sequenced to establish the identity of this DNA amplicon. The sequence of the 600 -bp band had 99 to $100 \%$ identity with a specific genomic region of many $P$. aeruginosa strains (VRFPA04, accession number CP008739.2; B136-33, CP004061.1; NCGM 1984, AP014646.1; NCGM 1900, AP014622.1; NCGM2.S1, AP012280.1). This genomic region overlaps $w b j C$ and $w b j D$ genes, which encodes an $\mathrm{O} 11 \mathrm{O}$-antigen biosynthesis associated epimerase and an UDP-Nacetylglucosamine 2-epimerase, respectively. These enzymes are involved in the synthesis of UDP-N-acetyl-L-fucosamine, a precursor involved in the production of lipopolysaccharides (LPS) in P. aeruginosa serotype $\mathrm{O} 11$ (Kneidinger et al., 2003; Mulrooney et al., 2005).

To confirm that the $w b j C$ and $w b j D$ genes are specific to $P$. aeruginosa isolates from the cluster III, a PCR-based genotyping test was performed on all the $P$. aeruginosa isolates using a primer pair specific to $w b j C$ and $w b j D$ genes and another targeting the gyrB gene as positive control. While the $g y r B$ primer pair gave an amplicon for every isolates, only those of the cluster III allowed a positive PCR signal for the $w b j C$ and $w b j D$ genes confirming that these genes are only present in isolates of cluster III.

\section{DISCUSSION}

We investigated the genetic and phenotypic characteristics of environmental isolates of $P$. aeruginosa to determine their similarity with clinical isolates. This was the first time that a large number of environmental isolates from dental unit waterlines were compared using RAPD and multiple phenotypic tests, including an amoeba predation assay. To determine whether there was a marked evolution of the bacteria in the lungs of CF patients as previously proposed (Kresse et al., 2003; Smith et al., 2006), we also analyzed clinical isolates from young CF patients between 4 and 18 years of age. We anticipated that the CF isolates would be genetically and phenotypically similar to the bacteria that had initially infected these patients.

We used RAPD to classify the $P$. aeruginosa isolates into clusters. We used three primers generating amplification patterns with a strong discriminatory potential. All the clinical isolates except two could be grouped into one cluster (cluster I). Since all the CF patients in the present study were treated in the same clinic, it was assumed that they would have harbored a limited number of strains, including variants, during the sampling period. On the other hand, we looked for relatively common, stable genetic characteristics in clinical isolates. The marked genetic similarity among the $P$. aeruginosa isolates collected over a 3-year period from the same patient (VD isolates; Table 1) showed that the isolates were relatively genetically stable. However, subtle changes (point mutations) in the genome of the isolates that can occur over a 3-year period might not have been detected by RAPD.

The clinical strains displayed greater variability than the environmental isolates in the phenotypic assays. Phenotypic variability of this kind has been reported previously (Deligianni et al., 2010). $P$. aeruginosa strains in CF lungs are subject to considerable selection pressure, which can increase the need of the strains to adapt through increased mutation rates, a phenomenon called the insurance effect (Boles et al., 2004).

A striking feature of our RAPD analysis was that environmental isolates could be segregated into two clusters. Interestingly, these two clusters displayed different phenotypic characteristics. Table 6 
Table 5 | Lysis of Dictyostelium discoideum cells exposed to $P$. aeruginosa culture supernatants.

\begin{tabular}{llll}
\hline$P$ aeruginosa isolates Clusters & $\begin{array}{l}\text { Average } \\
\text { (\% of cells) }\end{array}$ & SD Outcome $^{\mathrm{a}}$ \\
\hline
\end{tabular}

\begin{tabular}{|c|c|c|c|c|}
\hline VD 706 & I & 107.4 & 1.1 & $N$ \\
\hline VD 329 & । & 41.1 & 38.8 & V \\
\hline 297 & । & 62.5 & 47.3 & V \\
\hline VD 564 & । & 111.3 & 22.1 & V \\
\hline VD 171 & । & 81.7 & 28.2 & V \\
\hline VD 609 & I & 100.3 & 16.0 & $\mathrm{~N}$ \\
\hline 403 & 1 & 23.1 & 44.9 & V \\
\hline 585 & । & 109.8 & 3.6 & $\mathrm{~N}$ \\
\hline 359 & । & 91.9 & 61.2 & V \\
\hline$E-500$ & । & 78.2 & 49.3 & V \\
\hline 392 & 1 & 5.6 & 11.2 & $\mathbf{L}$ \\
\hline 279 & । & 94.9 & 21.2 & V \\
\hline 358 & 1 & 62.0 & 63.6 & V \\
\hline $578-A$ & । & 97.5 & 7.6 & $\mathrm{~N}$ \\
\hline PPF 18 & $\|$ & 6.1 & 10.2 & $\mathbf{L}$ \\
\hline Ortho 1 & $\|$ & 14.0 & 27.1 & V \\
\hline Chir D-144 assistant & $\|$ & 0.5 & 0.7 & $\mathbf{L}$ \\
\hline Urg 7 & $\|$ & 5.2 & 10.5 & $\mathbf{L}$ \\
\hline Urg 5 & $\|$ & 30.1 & 48.1 & V \\
\hline PPF 19 & $\|$ & 9.5 & 17.3 & $\mathbf{L}$ \\
\hline PPF 20 & $\|$ & 6.7 & 12.4 & $\mathbf{L}$ \\
\hline PPF 13 & $\|$ & 13.6 & 8.8 & $\mathbf{L}$ \\
\hline $578-B$ & $\|$ & 111.6 & 2.4 & $N$ \\
\hline 506 & $\|$ & 62.8 & 57.1 & V \\
\hline PPF2 & III & 107.6 & 9.2 & $N$ \\
\hline PPF1 & III & 94.9 & 12.9 & $N$ \\
\hline PPF21 & III & 96.0 & 3.5 & $N$ \\
\hline PPF7 & III & 113.0 & 6.7 & $\mathrm{~N}$ \\
\hline Chir D-144 & III & 105.2 & 2.9 & $\mathrm{~N}$ \\
\hline
\end{tabular}

The values are the average and SD of four independent experiments.

${ }^{a} L$, lysis; $N$, no lysis (or no significant lysis); $V$, variable.

presents a summary of the phenotypic trends observed in each cluster. The environmental isolates in cluster III displayed divergent and even opposite phenotypes compared to the isolates in the other two clusters. This is especially remarkable for the mucoid, biofilm formation, and capacity to lyse amoebae phenotypes. The environmental isolates in cluster II displayed phenotypes that were more similar to the clinical isolates. Genetic similarities based on RAPD results between clusters I and II could explain the phenotypes observed. In this regard, all the phenotypic differences observed between the cluster III and other isolates were in agreement with those reported in previous publications (Rocchetta etal., 1999; Smith etal., 2006). For example, the mucoid clinical $P$. aeruginosa strains from a patient with a chronic pulmonary infection, had a lower capacity to produce an adherent biofilm, and were less virulent. It appears that virulence factors are recognized by the immune system and that bacteria presenting virulence factors are eliminated. B-band LPS, which are the predominant polysaccharides of non-mucoid strains such as cluster III isolates, are anionic and may play a key role in binding and may thus promote the formation of a more adherent biofilm, as was observed with cluster III isolates.

Our analysis revealed that the dental unit waterlines contained two distinct populations of $P$. aeruginosa. It has been previously shown that dental unit waterlines are colonized by biofilms where opportunistic waterborne pathogens can proliferate (Barbeau et al., 1997; Walker et al., 2000). Earlier studies at Université de Montréal have shown that $P$. aeruginosa has a non-random distribution in these dental units since $89.5 \%$ of the isolates were isolated from only four of the nine clinics at the Faculty of Dentistry (Barbeau etal., 1996). There was no physical link between the dental units apart from the fact that they were all connected to the same water source (municipal drinking water). It was not possible to establish an association between a given dental unit and a given cluster. Since the environmental isolates from cluster III formed abundant biofilms, without being mucoid, these isolates may have originated from biofilms in the dental unit waterlines. The environmental isolates in cluster II, which did not form substantial biofilms in vitro, may have come from planktonic populations, which were transiently present in the dental unit waterlines.

The segregation of the environmental isolates into two distinct clusters revealed a number of interesting features. Strains in cluster III produced significantly more biofilm, displayed less elastase activity, and were unable to lyse amoebal cells despite the fact that their resistance to predation was not significantly different from that of the isolates in clusters I and II. This suggested that other factors that were not tested in the present study are involved in the interaction between $P$. aeruginosa and $D$. discoideum.

The predation resistance of the $P$. aeruginosa isolates of various origins has been tested using $D$. discoideum as the predator in four previous studies (Bradbury et al., 2011; Lelong et al., 2011; Janjua et al., 2012; Jeukens et al., 2014). P. aeruginosa strains isolated from CF patients of all ages (babies to adults) have been studied, including isolates that can spread to other patients like the Liverpool epidemic strain (LES). Isolates from urinary tract infections, skin ulcers, intubated patients, and the environment have also been tested using the $D$. discoideum predation assay. Even though the studies used different versions of the predation assay in which the amoeba strains, media, or scoring parameters were different, general conclusions can be drawn. Most of the $P$. aeruginosa isolates tested were resistant to $D$. discoideum predation. Half the isolates from CF teenagers, most isolates from CF adults, and all the transmissible CF isolates displayed little or no resistance to amoeba predation. Some authors suggested that the virulence of $P$. aeruginosa decreases when it adapts to the CF lung environment over a long period of time (Smith et al., 2006; Friman et al., 2013). In this study, we analyzed $P$. aeruginosa isolates from young CF patients. All the isolates, except two, were highly resistant to amoeba predation and produced high levels of elastase, 
Table 6 | Phenotypic trends of the three clusters ${ }^{a}$.

\begin{tabular}{|c|c|c|c|c|c|c|}
\hline Cluster & Mucoid phenotype & Biofilm production & Elastase activity & Predation resistance & Amoeba lysis & Epimerase genes \\
\hline I & ++ (variable) & + (variable) & +++ & +++ & Highly variable & - \\
\hline III & - & +++ & + & +++ & - & + \\
\hline
\end{tabular}

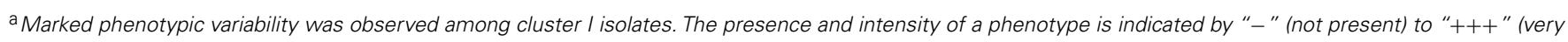
intense).

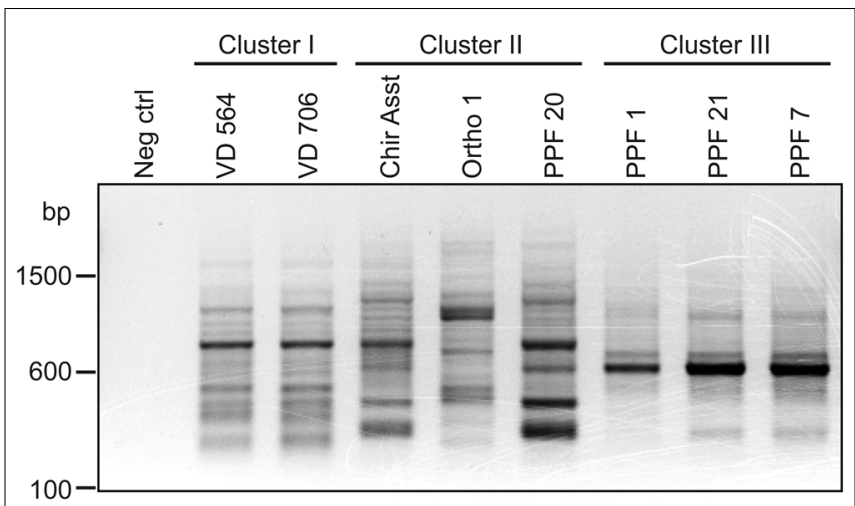

FIGURE 6 | Agarose gel electrophoresis of RAPD amplification products generated by the OPA-10 primer. Neg control refers to the negative control (no template DNA).

suggesting that they had not begun the adaptation-associated loss of virulence process. Our environmental isolates were resistant to $D$. discoideum predation, which is in agreement with previously published predation results for other environmental isolates (Bradbury et al., 2011). It thus appears that the predation assay is not the most sensitive assay for discriminating $P$. aeruginosa isolates of various origins. On the other hand, the cell lysing capacity of the isolates was a more useful parameter that made it possible to segregate the dental unit waterline isolates into two clusters based on their interactions with amoebae.

It is important to remember that the predation assay measures the cumulative effect of the entire arsenal deployed by a bacterium to resist predation by amoebae. However, it is possible that different "weapons" in the bacterial arsenal can result in the same level of resistance. For example, the cytolytic potential of cluster I and II isolates and the increased production of biofilms by cluster III isolates are different "weapons," but are both potentially efficient ways to resist amoeba predation.

Our molecular analyses showed that cluster III isolates are the only ones harboring the $w b j C$ and wbjD genes, which encode epimerases. As mentioned earlier, these enzymes are required for the synthesis of LPS. To our knowledge, no studies have investigated these epimerases to determine their involvement in biofilm formation. However, the contribution of other epimerases in biofilm formation has been studied in other bacterial species. For example, the galE gene that encodes UDP-galactose-4-epimerase is essential for the formation of biofilms by Xanthomonas campestris pv. campestris and Vibrio cholerae (Nesper etal., 2001; Li et al.,
2014), while the overexpression of the galE gene by Thermus thermophilus results in an increase in the production of biofilms (Niou et al., 2009). Surprisingly, the inactivation of the galE gene in Haemophilus parasuis and Porphyromonas gingivalis has the opposite effect, with higher biofilm production in mutant strains than in the wild-type bacteria (Nakao et al., 2006; Zou et al., 2013). In addition, the inactivation of ADP-glycero-manno-heptose 6epimerase, which is encoded by GmhD in Vibrio vulnificus, results in a defect in the production of mature LPS, attenuating the ability to form a biofilm (Kim et al., 2007). While their effects are not always clear, epimerases do appear to play a role in biofilm formation.

Of all the isolates analyzed, cluster III isolates displayed the greatest ability to form biofilms. So far, we cannot conclude that the increase in biofilm production is linked to the $w b j C$ and $w b j D$ genes but this study stresses the need to further invest this finding. Above all, it shows that the RAPD technique is a valuable approach for providing molecular insights into differences between various groups of isolates.

It is also interesting that the $w b j C$ and $w b j D$ genes are only found in a subset of strains/isolates, both in our study and in various genomic databases. For example, GenBank lists 301 P. aeruginosa genome assemblies, 40 of which have the 600-bp sequence specific to cluster III isolates that overlaps the $w b j C$ and $w b j D$ genes. The origins of the strains/isolates are rudimentarily indicated in the databases for 15 of the 40 genomes. These strains originated from infected corneas, community-acquired diarrhea, blood from a patient with an infection, urine from a patient with a urinary tract infection, sputum, gastric juice from a dolphin kept in captivity, a fuel tank, and a biofilm in an industrial water system. It is important to note that none of the origins are related to CF, further indicating that $P$. aeruginosa isolates harboring the $w b j C$ and $w b j D$ genes form a distinctive group that is likely not involved in chronic pulmonary infections.

\section{ACKNOWLEDGMENTS}

The authors thank Pierre Rompré for the statistical analyses. This work was supported by funding from Theratechnologies Inc. to Jean Barbeau and by grants to Steve J. Charette from the Chaire de pneumologie de la fondation J.-D. Bégin at Université Laval, the Fonds Alphonse L'Espérance de la fondation of IUCPQ, and the Natural Sciences and Engineering Research Council of Canada (NSERC). Myriam M. Ouellet received a scholarship from the Centre de recherche en infectiologie porcine et avicole (CRIPA). Christine Nadeau was supported by Burrows-Wellcome-MRC summer student scholarships and the Fonds de la Recherche en 
Santé du Québec. Special thanks to Dr. Stéphane Roy's team for assisting with the molecular biology procedures and cloning the epimerase gene.

\section{REFERENCES}

Barbeau, J., Avezard, C., Faucher, E., Zalzal, S., and Prévost, A. P. (1997). Biofilms in dental unit waterlines: ultrastructural and cytochemical analysis. Cell Mater. 7, 135-146.

Barbeau, J., Tanguay, R., Faucher, E., Avezard, C., Trudel, L., Cote, L., et al. (1996). Multiparametric analysis of waterline contamination in dental units. Appl. Environ. Microbiol. 62, 3954-3959.

Barbeau, J., Ten Bokum, L., Gauthier, C., and Prevost, A. P. (1998). Crosscontamination potential of saliva ejectors used in dentistry. J. Hosp. Infect. 40, 303-311. doi: 10.1016/S0195-6701(98)90308-5

Boles, B. R., Thoendel, M., and Singh, P. K. (2004). Self-generated diversity produces "insurance effects" in biofilm communities. Proc. Natl. Acad. Sci. U.S.A. 101, 16630-16635. doi: 10.1073/pnas.0407460101

Bradbury, R. S., Reid, D. W., Inglis, T. J., and Champion, A. C. (2011). Decreased virulence of cystic fibrosis Pseudomonas aeruginosa in Dictyostelium discoideum. Microbiol. Immunol. 55, 224-230. doi: 10.1111/j.1348-0421.2011. 00314.x

Chung, J. C., Becq, J., Fraser, L., Schulz-Trieglaff, O., Bond, N. J., Foweraker, J., et al. (2012). Genomic variation among contemporary Pseudomonas aeruginosa isolates from chronically infected cystic fibrosis patients. J. Bacteriol. 194, 48574866. doi: 10.1128/JB.01050-12

Cornillon, S., Pech, E., Benghezal, M., Ravanel, K., Gaynor, E., Letourneur, F., et al. (2000). Phglp is a nine-transmembrane protein superfamily member involved in Dictyostelium adhesion and phagocytosis. J. Biol. Chem. 275, 34287-34292. doi: 10.1074/jbc.M006725200

Cosson, P., Zulianello, L., Join-Lambert, O., Faurisson, F., Gebbie, L., Benghezal, M., et al. (2002). Pseudomonas aeruginosa virulence analyzed in a Dictyostelium discoideum host system. J. Bacteriol. 184, 3027-3033. doi: 10.1128/JB.184.11.30273033.2002

Deligianni, E., Pattison, S., Berrar, D., Ternan, N. G., Haylock, R. W., Moore, J. E., et al. (2010). Pseudomonas aeruginosa cystic fibrosis isolates of similar RAPD genotype exhibit diversity in biofilm forming ability in vitro. BMC Microbiol. 10:38. doi: 10.1186/1471-2180-10-38

Dettman, J. R., Rodrigue, N., Aaron, S. D., and Kassen, R. (2013). Evolutionary genomics of epidemic and nonepidemic strains of Pseudomonas aeruginosa. Proc. Natl. Acad. Sci. U.S.A. 110, 21065-21070. doi: 10.1073/pnas.1307 862110

Filion, G., and Charette, S. J. (2014). Assessing Pseudomonas aeruginosa virulence using a nonmammalian host: Dictyostelium discoideum. Methods Mol. Biol. 671680. doi: 10.1007/978-1-4939-0473-0_51

Fothergill, J. L., Mowat, E., Ledson, M. J., Walshaw, M. J., and Winstanley, C. (2010a). Fluctuations in phenotypes and genotypes within populations of Pseudomonas aeruginosa in the cystic fibrosis lung during pulmonary exacerbations. J. Med. Microbiol. 59, 472-481. doi: 10.1099/jmm.0.015875-0

Fothergill, J. L., White, J., Foweraker, J. E., Walshaw, M. J., Ledson, M. J., Mahenthiralingam, E., et al. (2010b). Impact of Pseudomonas aeruginosa genomic instability on the application of typing methods for chronic cystic fibrosis infections. J. Clin. Microbiol. 48, 2053-2059. doi: 10.1128/JCM. 00019-10

Friman, V. P., Ghoul, M., Molin, S., Johansen, H. K., and Buckling, A. (2013). Pseudomonas aeruginosa adaptation to lungs of cystic fibrosis patients leads to lowered resistance to phage and protist enemies. PLOS ONE 8:e75380. doi: 10.1371/journal.pone.0075380

Froquet, R., Lelong, E., Marchetti, A., and Cosson, P. (2009). Dictyostelium discoideum: a model host to measure bacterial virulence. Nat. Protoc. 4, 25-30. doi: 10.1038 /nprot.2008.212

Hafiane, A., and Ravaoarinoro, M. (2011). Characterization of Pseudomonas aeruginosa strains isolated from cystic fibrosis patients by different typing methods. Pathol. Biol. 59, e109-e114. doi: 10.1016/j.patbio.2009.06.002

Harrison, F. (2007). Microbial ecology of the cystic fibrosis lung. Microbiology 153, 917-923. doi: 10.1099/mic.0.2006/004077-0

Hu, H., Harmer, C., Anuj, S., Wainwright, C. E., Manos, J., Cheney, J., et al. (2013). Type 3 secretion system effector genotype and secretion phenotype of longitudinally collected Pseudomonas aeruginosa isolates from young children diagnosed with cystic fibrosis following newborn screening. Clin. Microbiol. Infect. 19, 266-272. doi: 10.1111/j.1469-0691.2012.03770.x

Janjua, H. A., Segata, N., Bernabo, P., Tamburini, S., Ellen, A., and Jousson, O. (2012). Clinical populations of Pseudomonas aeruginosa isolated from acute infections show a wide virulence range partially correlated with population structure and virulence gene expression. Microbiology 158, 2089-2098. doi: 10.1099/mic.0.056689-0

Jeukens, J., Boyle, B., Kukavica-Ibrulj, I., Ouellet, M. M., Aaron, S. D., Charette, S. J., et al. (2014). Comparative genomics of isolates of a Pseudomonas aeruginosa epidemic strain associated with chronic lung infections of cystic fibrosis patients. PLoS ONE 9:e87611. doi: 10.1371/journal.pone.0087611

Kim, H. S., Lee, M. A., Chun, S. J., Park, S. J., and Lee, K. H. (2007). Role of NtrC in biofilm formation via controlling expression of the gene encoding an ADP-glycero-manno-heptose-6-epimerase in the pathogenic bacterium, Vibrio vulnificus. Mol. Microbiol. 63, 559-574. doi: 10.1111/j.1365-2958.2006. 05527.x

Kneidinger, B., O’Riordan, K., Li, J., Brisson, J. R., Lee, J. C., and Lam, J. S. (2003). Three highly conserved proteins catalyze the conversion of UDP-N-acetyl-Dglucosamine to precursors for the biosynthesis of $\mathrm{O}$ antigen in Pseudomonas aeruginosa $\mathrm{O} 11$ and capsule in Staphylococcus aureus type 5. Implications for the UDP-N-acetyl-L-fucosamine biosynthetic pathway. J. Biol. Chem. 278, 36153627. doi: 10.1074/jbc.M203867200

Kresse, A. U., Dinesh, S. D., Larbig, K., and Römling, U. (2003). Impact of large chromosomal inversions on the adaptation and evolution of Pseudomonas aeruginosa chronically colonizing cystic fibrosis lungs. Mol. Microbiol. 47, 145-158. doi: 10.1046/j.1365-2958.2003.03261.x

Lelong, E., Marchetti, A., Simon, M., Burns, J. L., Van Delden, C., Köhler, T., et al. (2011). Evolution of Pseudomonas aeruginosa virulence in infected patients revealed in a Dictyostelium discoideum host model. Clin. Microbiol. Infect. 17, 1415-1420. doi: 10.1111/j.1469-0691.2010.03431.X

Li, C. T., Liao, C. T., Du, S. C., Hsiao, Y. P., Lo, H. H., and Hsiao, Y. M. (2014). Functional characterization and transcriptional analysis of galE gene encoding a UDP-galactose 4-epimerase in Xanthomonas campestris pv. campestris. Microbiol. Res. 169, 441-452. doi: 10.1016/j.micres.2013.08.005

Martin, M. V. (1987). The significance of the bacterial contamination of dental unit water systems. Br. Dent. J. 163, 152-154. doi: 10.1038/sj.bdj.4806220

Marvig, R. L., Johansen, H. K., Molin, S., and Jelsbak, L. (2013). Genome analysis of a transmissible lineage of Pseudomonas aeruginosa reveals pathoadaptive mutations and distinct evolutionary paths of hypermutators. PLoS Genet. 9:e1003741. doi: 10.1371/journal.pgen.1003741

Mereghetti, L., Marquet-van der Mee, N., Loulergue, J., Rolland, J. C., and Audurier, A. (1998). Pseudomonas aeruginosa from cystic fibrosis patients: study using whole cell RAPD and antibiotic susceptibility. Pathol. Biol. (Paris) 46, 319-324.

Mowat, E., Paterson, S., Fothergill, J. L., Wright, E. A., Ledson, M. J., Walshaw, M. J., et al. (2011). Pseudomonas aeruginosa population diversity and turnover in cystic fibrosis chronic infections. Am. J. Respir. Crit. Care Med. 183, 1674-1679. doi: 10.1164/rccm.201009-1430OC

Mulrooney, E. F., Poon, K. H., McNally, D. J., Brisson, J. R., and Lam, J. S. (2005). Biosynthesis of UDP-N-acetyl-L-fucosamine, a precursor to the biosynthesis of lipopolysaccharide in Pseudomonas aeruginosa serotype O11. J. Biol. Chem. 280, 19535-19542. doi: 10.1074/jbc.M500612200

Murray, T. S., Egan, M., and Kazmierczak, B. I. (2007). Pseudomonas aeruginosa chronic colonization in cystic fibrosis patients. Curr. Opin. Pediatr. 19, 83-88. doi: 10.1097/MOP.0b013e3280123a5d

Nakao, R., Senpuku, H., and Watanabe, H. (2006). Porphyromonas gingivalis galE is involved in lipopolysaccharide $\mathrm{O}$-antigen synthesis and biofilm formation. Infect. Immun. 74, 6145-6153. doi: 10.1128/IAI.00261-06

Nesper, J., Lauriano, C. M., Klose, K. E., Kapfhammer, D., Kraiss, A., and Reidl, J. (2001). Characterization of Vibrio cholerae O1 El tor galU and galE mutants: influence on lipopolysaccharide structure, colonization, and biofilm formation. Infect. Immun. 69, 435-445. doi: 10.1128/IAI.69.1.435-445.2001

Niou, Y. K., Wu, W. L., Lin, L. C., Yu, M. S., Shu, H. Y., Yang, H. H., et al. (2009). Role of galE on biofilm formation by Thermus spp. Biochem. Biophys. Res. Commun. 390, 313-318. doi: 10.1016/j.bbrc.2009.09.120

O’Donnell, M. J., Boyle, M. A., Russell, R. J., and Coleman, D. C. (2011). Management of dental unit waterline biofilms in the 21st century. Future Microbiol. 6, 1209-1226. doi: 10.2217/fmb.11.104 
Ortiz-Herrera, M., Geronimo-Gallegos, A., Cuevas-Schacht, F., Perez-Fernandez, L., and Coria-Jimenez, R. (2004). RAPD-PCR characterization of Pseudomonas aeruginosa strains obtained from cystic fibrosis patients. Salud Publica Mex. 46, 149-157. doi: 10.1590/S0036-36342004000200009

Podbielska, A., Galkowska, H., Stelmach, E., Mlynarczyk, G., and Olszewski, W. L. (2010). Slime production by Staphylococcus aureus and Staphylococcus epidermidis strains isolated from patients with diabetic foot ulcers. Arch. Immunol. Ther. Exp. (Warsz.) 58, 321-324. doi: 10.1007/s00005-010-0079-9

Rau, M. H., Marvig, R. L., Ehrlich, G. D., Molin, S., and Jelsbak, L. (2012). Deletion and acquisition of genomic content during early stage adaptation of Pseudomonas aeruginosa to a human host environment. Environ. Microbiol. 14, 2200-2211. doi: 10.1111/j.1462-2920.2012.02795.x

Rocchetta, H. L., Burrows, L. L., and Lam, J. S. (1999). Genetics of O-antigen biosynthesis in Pseudomonas aeruginosa. Microbiol. Mol. Biol. Rev. 63, 523-553.

Rust, L., Messing, C. R., and Iglewski, B. H. (1994). Elastase assays. Methods Enzymol. 235, 554-562. doi: 10.1016/0076-6879(94)35170-8

Schmidt, K. D., Tummler, B., and Römling, U. (1996). Comparative genome mapping of Pseudomonas aeruginosa $\mathrm{PAO}$ with $P$. aeruginosa $\mathrm{C}$, which belongs to a major clone in cystic fibrosis patients and aquatic habitats. J. Bacteriol. 178, 85-93.

Singh, P. K., Schaefer, A. L., Parsek, M. R., Moninger, T. O., Welsh, M. J., and Greenberg, E. P. (2000). Quorum-sensing signals indicate that cystic fibrosis lungs are infected with bacterial biofilms. Nature 407, 762-764. doi: 10.1038/35037627 Smith, E. E., Buckley, D. G., Wu, Z., Saenphimmachak, C., Hoffman, L. R., D’argenio, D. A., et al. (2006). Genetic adaptation by Pseudomonas aeruginosa to the airways of cystic fibrosis patients. Proc. Natl. Acad. Sci. U.S.A. 103, 8487-8492. doi: 10.1073/pnas.0602138103

Smith, G. L., Socransky, S. S., and Smith, C. M. (1989). Rapid method for the purification of DNA from subgingival microorganisms. Oral Microbiol. Immunol. 4, 47-51. doi: 10.1111/j.1399-302X.1989.tb00406.x

Stehling, E. G., Leite, D. S., and Silveira, W. D. (2010). Molecular typing and biological characteristics of Pseudomonas aeruginosa isolated from cystic fibrosis patients in Brazil. Braz. J. Infect. Dis. 14, 462-467. doi: 10.1016/S1413-8670(10)70094-5
Walker, J. T., Bradshaw, D. J., Bennett, A. M., Fulford, M. R., Martin, M. V., and Marsh, P. D. (2000). Microbial biofilm formation and contamination of dentalunit water systems in general dental practice. Appl. Environ. Microbiol. 66, 33633367. doi: 10.1128/AEM.66.8.3363-3367.2000

Wilson, K. (2001). Preparation of genomic DNA from bacteria. Curr. Protoc. Mol. Biol. Chap. 2, Unit 2.4. doi: 10.1002/0471142727.mb0204s56

Workentine, M. L., Sibley, C. D., Glezerson, B., Purighalla, S., Norgaard-Gron, J. C., Parkins, M. D., et al. (2013). Phenotypic heterogeneity of Pseudomonas aeruginosa populations in a cystic fibrosis patient. PLoS ONE 8:e60225. doi: 10.1371/journal.pone.0060225

Zou, Y., Feng, S., Xu, C., Zhang, B., Zhou, S., Zhang, L., et al. (2013). The role of galU and galE of Haemophilus parasuis SC096 in serum resistance and biofilm formation. Vet. Microbiol. 162, 278-284. doi: 10.1016/j.vetmic.2012.08.006

Conflict of Interest Statement: The authors declare that the research was conducted in the absence of any commercial or financial relationships that could be construed as a potential conflict of interest.

Received: 06 November 2014; accepted: 29 December 2014; published online: 21 January 2015.

Citation: Ouellet MM, Leduc A, Nadeau C, Barbeau J and Charette SJ (2015) Pseudomonas aeruginosa isolates from dental unit waterlines can be divided in two distinct groups, including one displaying phenotypes similar to isolates from cystic fibrosis patients. Front. Microbiol. 5:802. doi: 10.3389/fmicb.2014.00802

This article was submitted to Infectious Diseases, a section of the journal Frontiers in Microbiology.

Copyright (c) 2015 Ouellet, Leduc, Nadeau, Barbeau and Charette. This is an openaccess article distributed under the terms of the Creative Commons Attribution License (CC BY). The use, distribution or reproduction in other forums is permitted, provided the original author(s) or licensor are credited and that the original publication in this journal is cited, in accordance with accepted academic practice. No use, distribution or reproduction is permitted which does not comply with these terms. 